Supporting Information

\title{
Carbon Nanotubes Readily Disperse in Linear Silicones and Improve the Thermal Stability of Dimethylsilicone Elastomers
}

Daniel H. Flagg, Thomas J. McCarthy*,
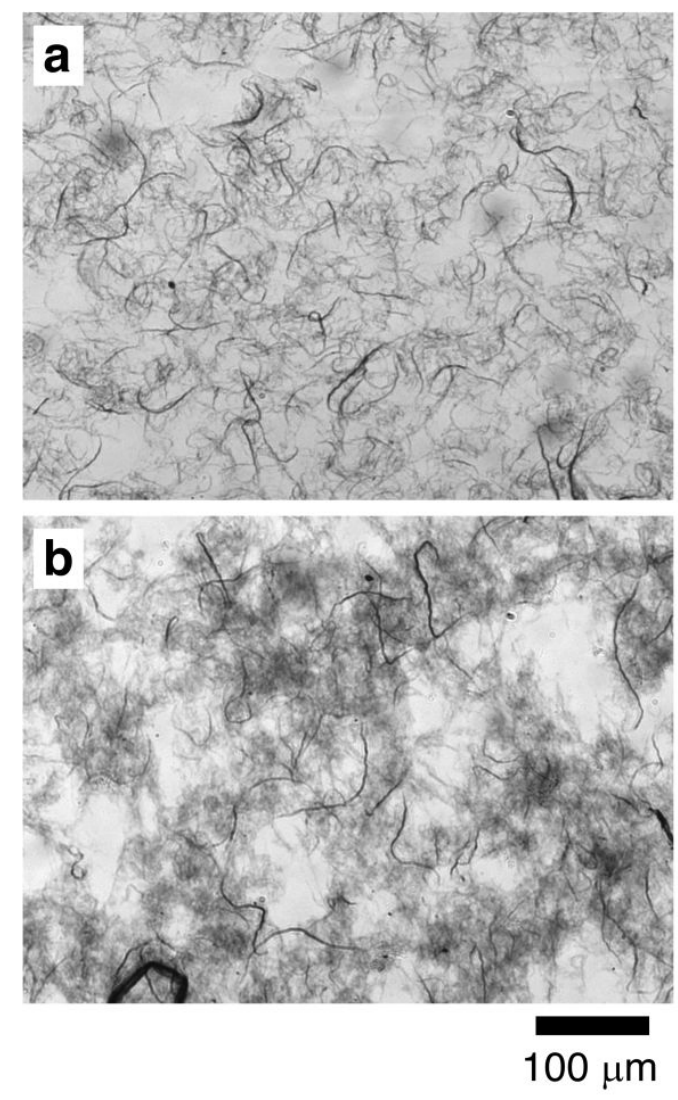

Figure S1. Optical micrographs (20x) of $0.1 \mathrm{wt} \%$ MWCNT/copolymer silicone fluid dispersions:

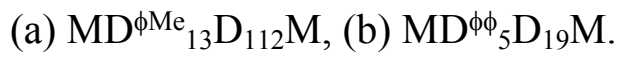



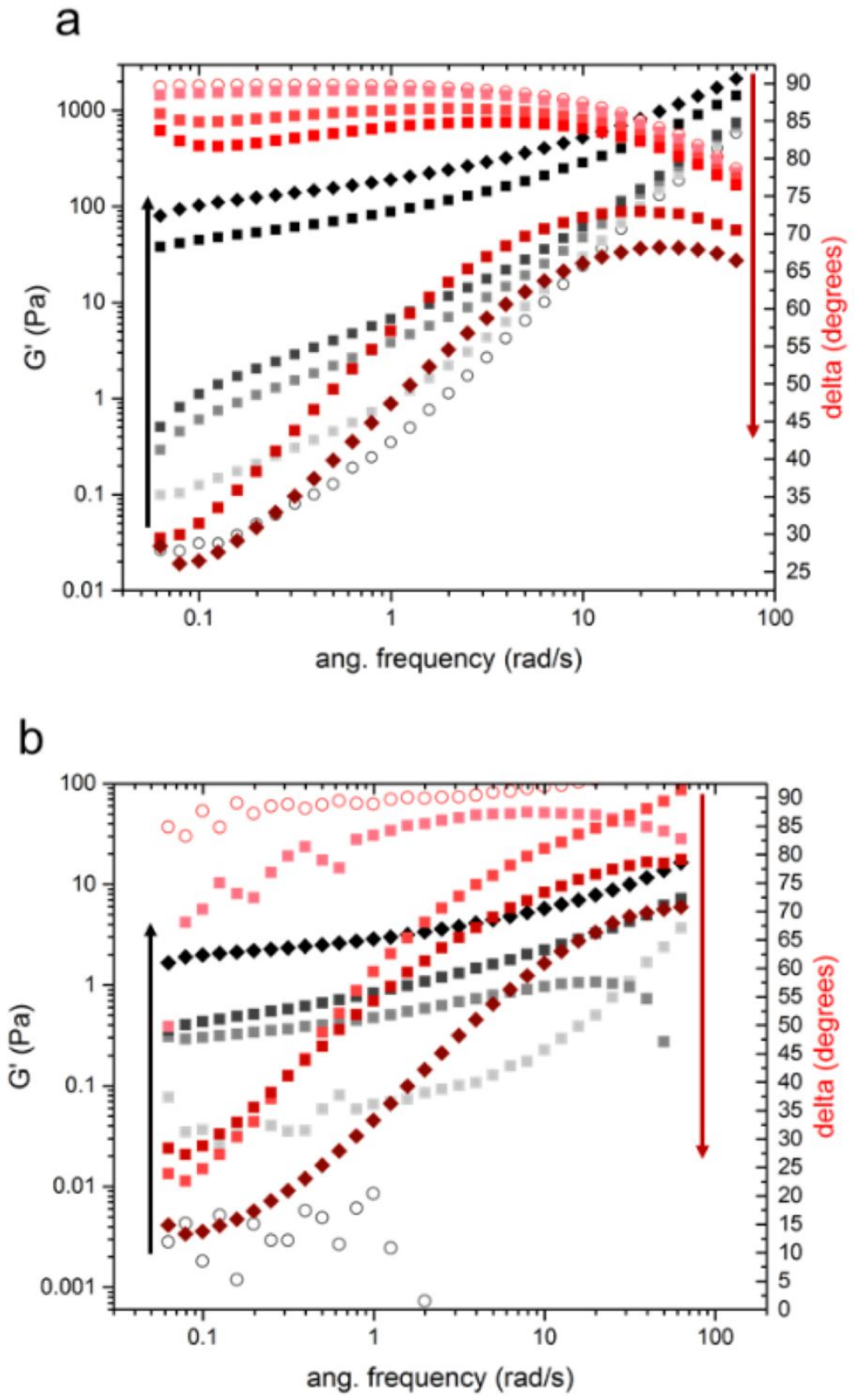

Figure S2. Oscillatory frequency sweeps at room temperature of (a) $\mathrm{MD}_{1570} \mathrm{M}$ dispersions and (b) $\mathrm{MD}_{230} \mathrm{M}$ dispersions. Arrows indicate increasing CNT concentration. Unfilled control samples are represented as unfilled circles. In (a) increasing symbol intensity corresponds to loadings of $0.01,0.05,0.1,0.5$, and $1.0 \mathrm{wt} \%$ CNT. In (b) increasing symbol intensity corresponds to loadings of $0.01,0.025,0.05$ and $0.1 \mathrm{wt} \% \mathrm{CNT}$. In general, G' increases and delta decreases with CNT loading. All experiments were run in the linear viscoelastic region. 
Table S1. Surface Resistivity of Silicone-CNT Elastomers $(\Omega / \square)$

\begin{tabular}{lllll}
\multicolumn{2}{l}{ Vinyl Component } & wt \% CNT & & \\
& $\mathbf{0 . 0 1}$ & $\mathbf{0 . 0 2 5}$ & $\mathbf{0 . 0 5}$ & $\mathbf{0 . 1}$ \\
\hline $\mathrm{M}^{\mathrm{V}} \mathrm{D}_{230} \mathrm{M}^{\mathrm{V}}$ & $3.77 \mathrm{E}+08$ & $1.31 \mathrm{E}+06$ & $1.10 \mathrm{E}+05$ & $4.58 \mathrm{E}+03$ \\
$\mathrm{M}^{\mathrm{V}} \mathrm{D}_{380} \mathrm{M}^{\mathrm{V}}$ & - & $8.90 \mathrm{E}+07$ & $1.65 \mathrm{E}+06$ & $6.95 \mathrm{E}+04$ \\
$\mathrm{PDMS}(\mathrm{OE} 41)$ & - & $1.60 \mathrm{E}+12$ & $7.56 \mathrm{E}+08$ & $4.58 \mathrm{E}+04$ \\
$\mathrm{M}^{\mathrm{V}} \mathrm{D}_{98} \mathrm{D}^{\phi \phi_{27}} \mathrm{M}^{\mathrm{V}}$ & $5.13 \mathrm{E}+09$ & $7.00 \mathrm{E}+06$ & $1.39 \mathrm{E}+06$ & $1.20 \mathrm{E}+05$ \\
$\mathrm{M}^{\mathrm{V}} \mathrm{D}_{100} \mathrm{D}^{\mathrm{Et}}{ }_{25} \mathrm{M}^{\mathrm{V}}$ & $8.00 \mathrm{E}+05$ & $1.14 \mathrm{E}+06$ & $8.00 \mathrm{E}+04$ & $2.40 \mathrm{E}+03$ \\
$\mathrm{M}^{\mathrm{V}} \mathrm{D}^{\mathrm{Et}}{ }_{58} \mathrm{M}^{\mathrm{V}}$ & $4.87 \mathrm{E}+04$ & $1.53 \mathrm{E}+05$ & $4.07 \mathrm{E}+03$ & $1.50 \mathrm{E}+03$ \\
\hline
\end{tabular}

Notes on Table S1. Elastomers were prepared with vinyl-terminated silicone fluids and hydridomethyl-dimethyl copolymers using $\sim 10$ ppm Karstedt's catalyst (2\% Pt solution in xylene, Gelest). Vinyl terminated silicone fluids purchased from Gelest were: polydimethylsiloxane (M 17 200, Gelest DMS-V25, $\mathrm{M}^{\mathrm{V}} \mathrm{D}_{230} \mathrm{M}^{\mathrm{V}}$ and $\mathrm{M} \sim 28000$, Gelest DMS$\mathrm{V} 31, \mathrm{M}^{\mathrm{V}} \mathrm{D}_{380} \mathrm{M}^{\mathrm{V}}$ ), poly(dimethyl-co-diethyl)siloxane (M 10 000, $20 \mathrm{~mol} \% \mathrm{D}^{\mathrm{Et}}$, Gelest EDV2022, $\mathrm{M}^{\mathrm{V}} \mathrm{D}_{100} \mathrm{D}^{\mathrm{Et}}{ }_{25} \mathrm{M}^{\mathrm{V}}$ ), poly(diphenyl-co-dimethyl)siloxane(M 12 500, $22 \mathrm{~mol} \%$ diphenyl, Gelest PDV-2331, ${ }^{\mathrm{V}} \mathrm{MD}_{98} \mathrm{D}^{\phi \phi_{27}} \mathrm{M}^{\mathrm{V}}$ ) and poly(phenylmethyl)siloxane (M 3000, Gelest PMV$9925, \mathrm{M}^{\mathrm{V}} \mathrm{D}^{\phi \mathrm{Me}}{ }_{22} \mathrm{M}^{\mathrm{V}}$ ) . Vinyl-terminated diethylsiloxane was prepared by equilibrium ringopening polymerization of cyclic diethylsiloxane oligomers (Gelest) using tetramethylammonium hydroxide as catalyst and divinyltetramethyldisiloxane as end-capper. The product has a molecular weight of $6000\left(\mathrm{M}^{\mathrm{V}} \mathrm{D}^{\mathrm{Et}}{ }_{58} \mathrm{M}^{\mathrm{V}}\right)$. Vinyl-containing siloxane polymers were crosslinked using a $30 \mathrm{~mol} \%$ hydridomethyl dimethyl copolymer (Gelest HMS-301, $\left.\mathrm{MD}_{18} \mathrm{D}^{\mathrm{H}}{ }_{8} \mathrm{M}\right)$ and $\sim 10 \mathrm{ppm}$ Pt catalyst. An additional entry in the table is the PDMS-based elastomer prepared from Gelest, OE41. The $\mathrm{M}^{\mathrm{V}} \mathrm{D}^{\phi \mathrm{Me}}{ }_{22} \mathrm{M}^{\mathrm{V}}$ system did not cure and could not be compared. 


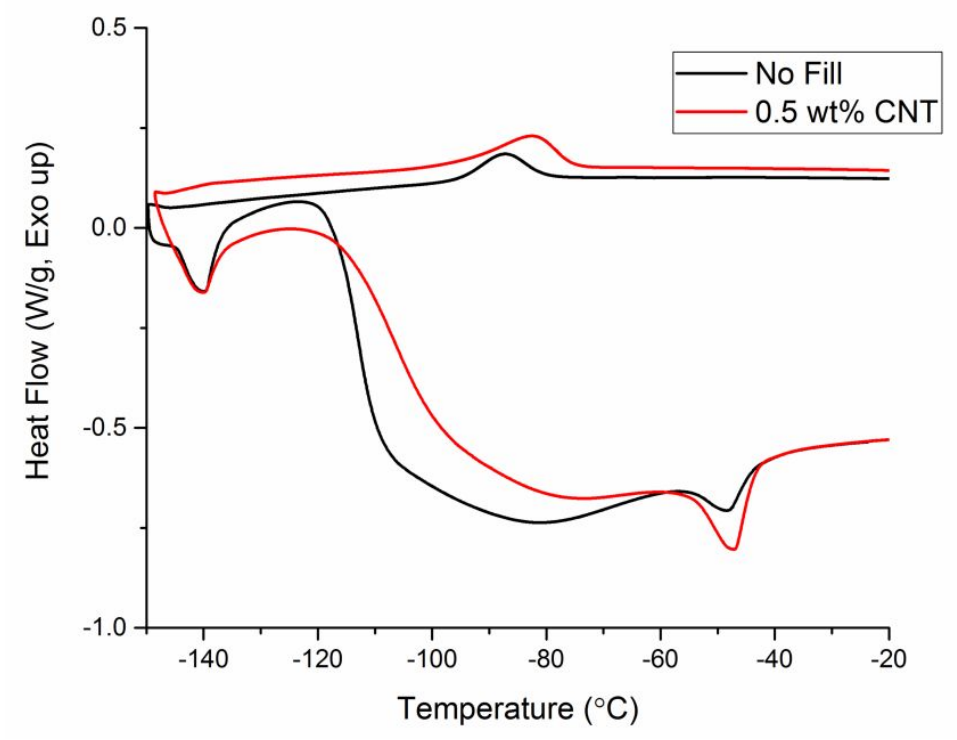

Figure S3. DSC (exotherm up) of unfilled and $0.5 \mathrm{wt} \%$ MWCNT composite elastomers.

Crystallization is observed in the cooling step (top curve) and melting is observed in the heating step (bottom curve). The glass transition is detected in the heating step (bottom curve) by the shift of the baseline indicating a change in heat capacity.

Table S2. DSC Glass Transition and Crystallization Data for $0.5 \mathrm{wt} \%$ MWCNT in PDMS Elastomer

\begin{tabular}{llllll} 
& $\mathbf{T}_{\mathbf{g}}$ & $\mathbf{T}_{\mathbf{c}, \text { heating }}$ & $\mathbf{T}_{\mathbf{c}, \text { cooling }}$ & $\mathbf{H}_{\mathbf{c}, \text { heating }}$ & $\mathbf{H}_{\mathbf{c}, \text { cooling }}$ \\
\hline No Filler & -121 & -47.6 & -86.1 & 10.18 & 14.27 \\
$0.5 \mathrm{wt} \%$ CNT & -112 & -47.1 & -82.6 & 8.877 & 12.46 \\
\hline
\end{tabular}

Notes on Figure S3 and Table S2. Differential scanning calorimetry (DSC) was conducted with a TA Q200 DSC with liquid nitrogen cooling. Samples were prepared in hermetically sealed aluminum pans and were heated to $150^{\circ} \mathrm{C}$ at $10^{\circ} \mathrm{C} / \mathrm{min}$, then cooled to $-150{ }^{\circ} \mathrm{C}$ at $10^{\circ} \mathrm{C} / \mathrm{min}$, and finally heated to 150 - at $10^{\circ} \mathrm{C} / \mathrm{min}$. 

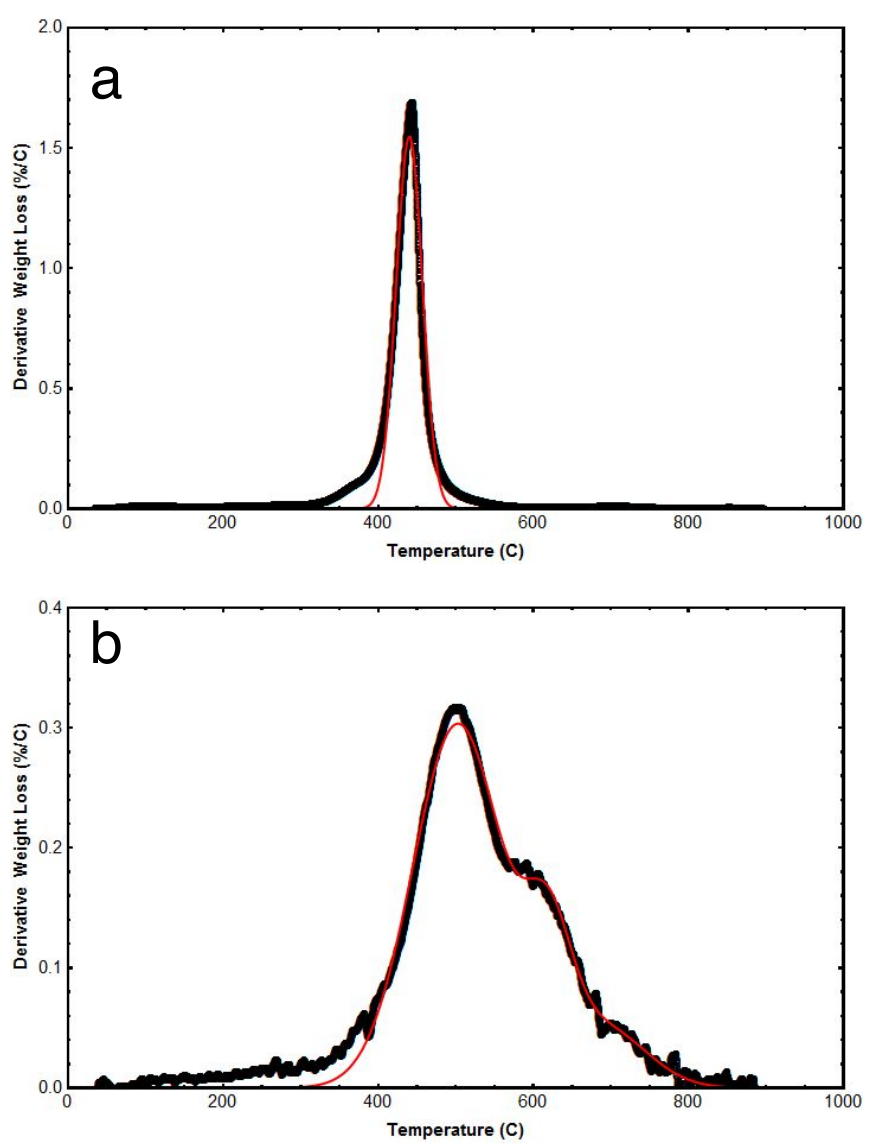

Figure S4. Examples of derivative weight loss (black) and total curve fit (red) from deconvolution into Gaussian functions for (a) an unfilled PDMS elastomer and (b) a $0.5 \mathrm{wt} \%$ MWCNT composite.

Table S3. Contributions of Degradation Processes Calculated from Gaussian Deconvolution for $0.5 \mathrm{wt} \%$ PDMS-MWCNT Composite Samples

$\begin{array}{cccccc}\text { Heating Rate } & \text { Peak 1, \% } & \text { Peak 2, \% } & \text { Peak 3, \% } & \text { Total Area } & \text { Char } \\ 1 & 86 & 4 & 10 & 53 & 43.7 \\ 2.5 & 89 & 2 & 9 & 53.5 & 44 \\ 5 & 87 & 3 & 10 & 54 & 42.8 \\ 10 & 78 & 10 & 12 & 54 & 40 \\ 20 & 75 & 13 & 12 & 59 & 37\end{array}$


Table S4. PCFC results for PDMS-MWCNT Composites

\begin{tabular}{cccccc} 
& $\begin{array}{c}\text { Peak Temp } \\
\left(\mathrm{TGA},{ }^{\circ} \mathrm{C}\right)\end{array}$ & $\begin{array}{c}\text { Char } \\
\left(\mathrm{TGA}, 900{ }^{\circ} \mathrm{C}\right)\end{array}$ & $\begin{array}{c}\text { Total HR } \\
(\mathrm{kJ} / \mathrm{g})\end{array}$ & $\begin{array}{c}\text { HRC } \\
(\mathrm{J} / \mathrm{g} \cdot \mathrm{K})\end{array}$ & $\begin{array}{c}\text { Peak Temp } \\
\left(\mathrm{PCFC},{ }^{\circ} \mathrm{C}\right)\end{array}$ \\
\hline Control & 445 & 0.19 & 18.8 & 538 & 466 \\
$0.1 \mathrm{wt} \% \mathrm{CNT}$ & 456 & 0.2 & 18.8 & 411 & 479 \\
$0.25 \mathrm{wt} \% \mathrm{CNT}$ & 470 & 0.3 & 17.8 & 207 & 493 \\
$0.5 \mathrm{wt} \% \mathrm{CNT}$ & 500 & 0.4 & 14.8 & 188 & 515 \\
\hline
\end{tabular}

Table S5. ${ }^{\text {Py } G C M S}$ results for PDMS-MWCNT Composites (duplicate runs of same samples)

\begin{tabular}{llllll}
\hline & $\begin{array}{l}\text { Pyrolysis } \\
\text { Temp. }\left({ }^{\circ} \mathbf{C}\right)\end{array}$ & D3 (\%) & D4 (\%) & Other (\%) & D3:D4 \\
\hline $\begin{array}{l}\text { PDMS } \\
\text { elastomer }\end{array}$ & 900 & 55 & 33.4 & 11.6 & 1.65 \\
& 900 & 56 & 33 & 11 & 1.70 \\
PDMS & 900 & 64 & 26 & 10.0 & 2.46 \\
$+.5 w t \%$ & 900 & 63.8 & 26.1 & 10.1 & 2.44 \\
MWCNT & & & & & \\
& 900 & 86.0 & 13.8 & $<0.5$ & 6.23 \\
MD $_{230} \mathrm{M}$ & 900 & 91.5 & 8.4 & $<0.5$ & 10.90 \\
& & & & & \\
\hline
\end{tabular}



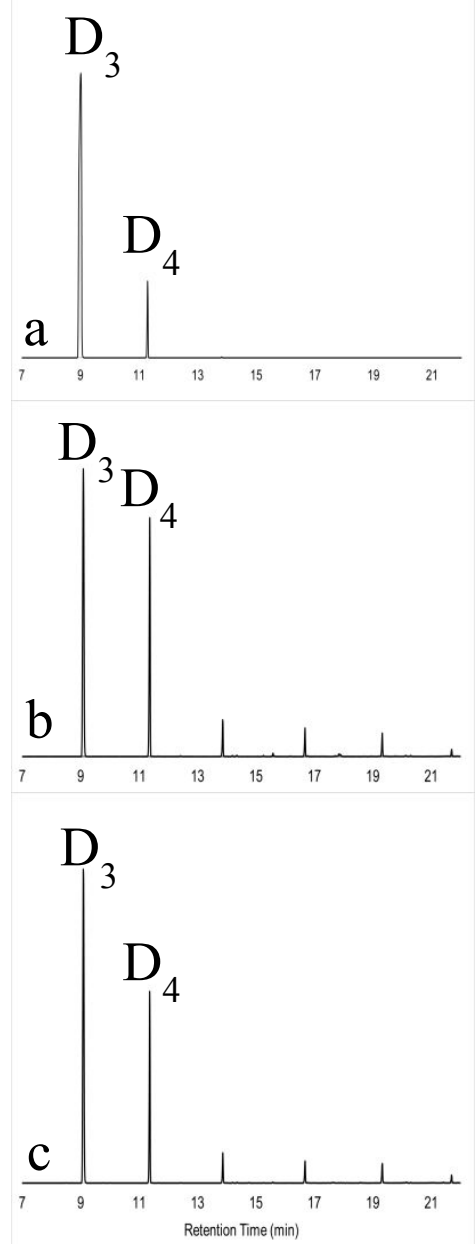

Figure S5. Chromatograms of ${ }^{\mathrm{Py}} \mathrm{GCMS}$ analysis for (a) $\mathrm{MD}_{230} \mathrm{M}$, (b) unfilled PDMS elastomer and (c) $0.5 \mathrm{wt} \%$ MWCNT-filled elastomer. 